\title{
Achieving ICME with Multiscale Modeling: The Effects of Constituent Properties and Processing on the Performance of Laminated Polymer Matrix Composite Structures
}

\author{
Evan J. Pineda, ${ }^{*}$ Brett A. Bednarcyk ${ }^{\dagger}$ and Steven M. Arnold $\ddagger$ \\ NASA Glenn Research Center, Cleveland, OH, 44135, USA
}

\begin{abstract}
Integrated computational materials engineering (ICME) is a useful approach for tailoring the performance of a material. For fiber-reinforced composites, not only do the properties of the constituents of the composite affect the performance, but so does the architecture (or microstructure) of the constituents. The generalized method of cells is demonstrated to be a viable micromechanics tool for determining the effects of the microstructure on the performance of laminates. The micromechanics is used to predict the inputs for a macroscale model for a variety of different fiber volume fractions, and fiber architectures. Using this technique, the material performance can be tailored for specific applications by judicious selection of constituents, volume fraction, and architectural arrangement given a particular manufacturing scenario.
\end{abstract}

\section{Nomenclature}

\begin{tabular}{ll}
$A, B$ & MMCDM damage stiffness parameters \\
$A^{C}, B^{C}$ & MMCDM compressive damage stiffness parameters \\
$A^{T}, B^{T}$ & MMCDM tensile damage stiffness parameters \\
$\mathbf{A}$ & Strain concentration matrix \\
$\mathbf{A}^{(\alpha \beta \gamma)}$ & Subcell strain concentration submatrix \\
$\mathbf{B}$ & Global RUC stiffness, Pa \\
$b_{i i}^{C}$ & Normal compressive damage weighting parameters \\
$b_{i i}^{T}$ & Tensie compressive damage weighting parameters \\
$b_{i j}$ & Damage weighting parameters \\
$\mathbf{C}^{(\alpha \beta \gamma)}$ & Subcell stiffness, Pa \\
$D, H, L$ & RUC dimensions, m \\
$D_{1}^{C}, D_{2}^{C}, D_{3}^{C}$ & MMCDM compressive scalar damage variables \\
$D_{1}^{T}, D_{2}^{T}, D_{3}^{T}$ & MMCDM tensile scalar damage variables \\
$D_{1}, D_{2}, D_{3}$ & MMCDM scalar damage variables \\
$d_{\alpha}, h_{\beta}, l_{\gamma}$ & Subcell dimensions, m \\
$d_{1}, d_{2}, d_{3}$ & MMCDM degradation factors \\
$d D_{i}$ & MMCDM damage increment \\
$d \epsilon_{i}^{D}$ & Damage strain increment \\
$E^{m}$ & Young's modulus of matrix, Pa \\
$E_{11}^{f}, E_{22}^{f}$ & Axial and transvese moduli of fiber, Pa \\
$E_{1}^{0}, E_{2}^{0}, E_{3}^{0}$ & Undamaged Young's moduli, Pa \\
\hline
\end{tabular}

*Aerospace Research Engineer, Mechanics and Life Prediction Branch, 21000 Brookpark Rd., MS 49-7, evan.j.pineda@nasa.gov, AIAA Member.

${ }^{\dagger}$ Materials Research Engineer, Mechanics and Life Prediction Branch, 21000 Brookpark Rd., MS 49-7, AIAA Associate Fellow.

${ }^{\ddagger}$ Branch Chief, Mechanics and Life Prediction Branch, 21000 Brookpark Rd., MS 49-7, AIAA Member. 


\begin{tabular}{|c|c|}
\hline$E_{1}, E_{2}, E_{3}$ & Young's moduli, Pa \\
\hline$E_{22}^{0}$ & Undamaged transverse modulus of lamina, $\mathrm{Pa}$ \\
\hline$E_{22}$ & Transverse modulus of lamina, $\mathrm{Pa}$ \\
\hline$e_{S}^{T}, e_{S}^{C}, g_{S}$ & Tensile, compressive, and shear ST microdamage functions \\
\hline$f$ & Thermodynamic driving force \\
\hline$G_{12}^{f}$ & Shear modulus of fiber, $\mathrm{Pa}$ \\
\hline$G_{12}^{0}, G_{13}^{0}, G_{23}^{0}$ & Undamaged shear moduli, $\mathrm{Pa}$ \\
\hline$G_{12}, G_{13}, G_{23}$ & Shear moduli, $\mathrm{Pa}$ \\
\hline$k_{i}$ & Instantaneous tangent stiffness, $\mathrm{Pa}$ \\
\hline$k_{i}^{\prime}$ & Normalized tangent stiffness \\
\hline$N_{\alpha}, N_{\beta}, N_{\gamma}$ & Total number of subcells \\
\hline$Q_{\epsilon}, R_{\epsilon}, S_{\epsilon}$ & Shear strain allowables \\
\hline$S$ & ST microdamage ISV/dissipated potential, $\mathrm{Pa}$ \\
\hline$\dot{S}$ & Rate ST microdamage ISV/dissipated potential, Pa/s \\
\hline$S_{r}$ & ST reduced microdamage ISV, $\mathrm{Pa}^{\frac{1}{3}}$ \\
\hline$W$ & Elastic strain energy density, Pa \\
\hline$W_{T}$ & Total work potential, $\mathrm{Pa}$ \\
\hline$X_{\epsilon}^{C}, Y_{\epsilon}^{C}, Z_{\epsilon}^{C}$ & Compressive normal strain allowables \\
\hline$X_{\epsilon}^{T}, Y_{\epsilon}^{T}, Z_{\epsilon}^{T}$ & Tensile normal strain allowables \\
\hline$X_{\epsilon}, Y_{\epsilon}, Z_{\epsilon}$ & Normal strain allowables \\
\hline$x_{1}, x_{2}, x_{3}$ & Macroscale composite coordinates \\
\hline$y_{1}, y_{2}, y_{3}$ & Microscale RUC coordinates \\
\hline$\alpha, \beta, \gamma$ & Subcell indices \\
\hline$\gamma_{12}, \gamma_{13}, \gamma_{23}$ & Engineering shear strain components \\
\hline $\bar{\epsilon}$ & Average global strains \\
\hline$\epsilon_{S}$ & Vector containing all Subcell strains \\
\hline $\boldsymbol{\epsilon}^{(\alpha \beta \gamma)}$ & Subcell strains \\
\hline$\epsilon_{1}^{D}, \epsilon_{2}^{D}, \epsilon_{3}^{D}$ & Damage strains \\
\hline$\epsilon_{11}, \epsilon_{22}, \epsilon_{33}$ & Normal strain components \\
\hline$\nu^{m}$ & Poisson's ratio of matrix \\
\hline$\nu_{12}^{f}, \nu_{23}^{f}$ & Axial and transverse Poisson's ratios of fiber \\
\hline$\nu_{12}^{0}, \nu_{13}^{0}, \nu_{21}^{0}, \nu_{23}^{0}, \nu_{31}^{0}, \nu_{32}^{0}$ & Undamaged Poisson's ratios \\
\hline$\nu_{12}, \nu_{13}, \nu_{21}, \nu_{23}, \nu_{31}, \nu_{32}$ & Poisson's ratios \\
\hline$\sigma_{i}^{D}$ & Damage stress, $\mathrm{Pa}$ \\
\hline $\boldsymbol{\sigma}^{(\alpha \beta \gamma)}$ & Subcell stresses $\mathrm{Pa}$ \\
\hline$\sigma_{11}, \sigma_{22}, \sigma_{33}$ & Normal stress components, $\mathrm{Pa}$ \\
\hline
\end{tabular}

\section{Introduction}

With the increased emphasis on reducing the cost and time to market of new materials, Integrated Computational Materials Engineering (ICME) has become a fast growing discipline within materials science and engineering. ICME is an integrated approach to the design of products and the materials which comprise them by linking material models at multiple time and length scales; such that manufacturing processes, which produce internal material structures that in turn influence material properties and allowables, can be tailored (engineered) to specific industrial applications. Figure 1 illustrates the interconnection of these scales and their cause/effect relationships; e.g., processing conditions produce a particular microstructure from which properties are obtained, which then dictate a specific structural performance. Note that the evolution of elliptical line types (i.e., dotted to dashed to solid line) are purposely included to visually suggest the level of maturity/understanding (from immature, to semi-mature, to mature, respectively) of modeling at each level of scale (both temporal and geometric). Furthermore, the figure demonstrates the importance of understanding the input and output at each scale in order to determine meaningful properties that are ultimately required by a structural analyst. Equally important is the fact that experiments (whether virtual or laboratory) performed at a given level can be viewed either as exploration or characterization experiments used to obtain the necessary model features or parameters, respectively, operating at the present and/or next 
higher level, or, they can be used to validate the modeling methods employed to transition from the lower level to the given level.

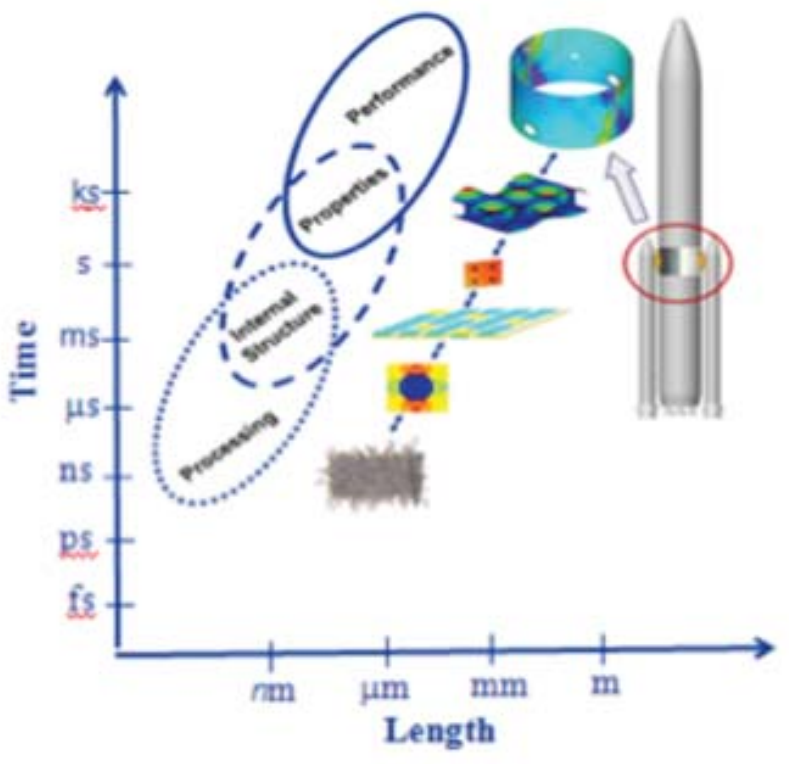

Figure 1: Schematic illustrating the multiple spatial and temporal scales active within material and structure.

While there is a clear indication that ICME is growing, the realization of the successful implementation of ICME in daily work of researchers and engineers in industry and academia is still lacking. In this paper we will demonstrate how the general, synergistic, multiscale-modeling framework for composites (developed by the NASA Glenn Research Center (GRC) and known as MAC/GMC and FEAMAC, see ${ }^{1}$ ) can be effectively utilized to link the material microstructure (e.g., constituent phase properties, volume fraction, fiber packing, etc.) to ply/laminate properties (mesoscale) and finally to performance (at the macroscale), see Figure 2, in an efficient and accurate manner to enable "fit-for-purpose" tailoring of the composite material. The ability to localize and homogenize between scales with efficiency and accuracy make MAC/GMC and FEAMAC ideal candidates for ICME simulations in a multiscale environment in which the microstructure can be optimized spatially based on the local loading and environmental history.

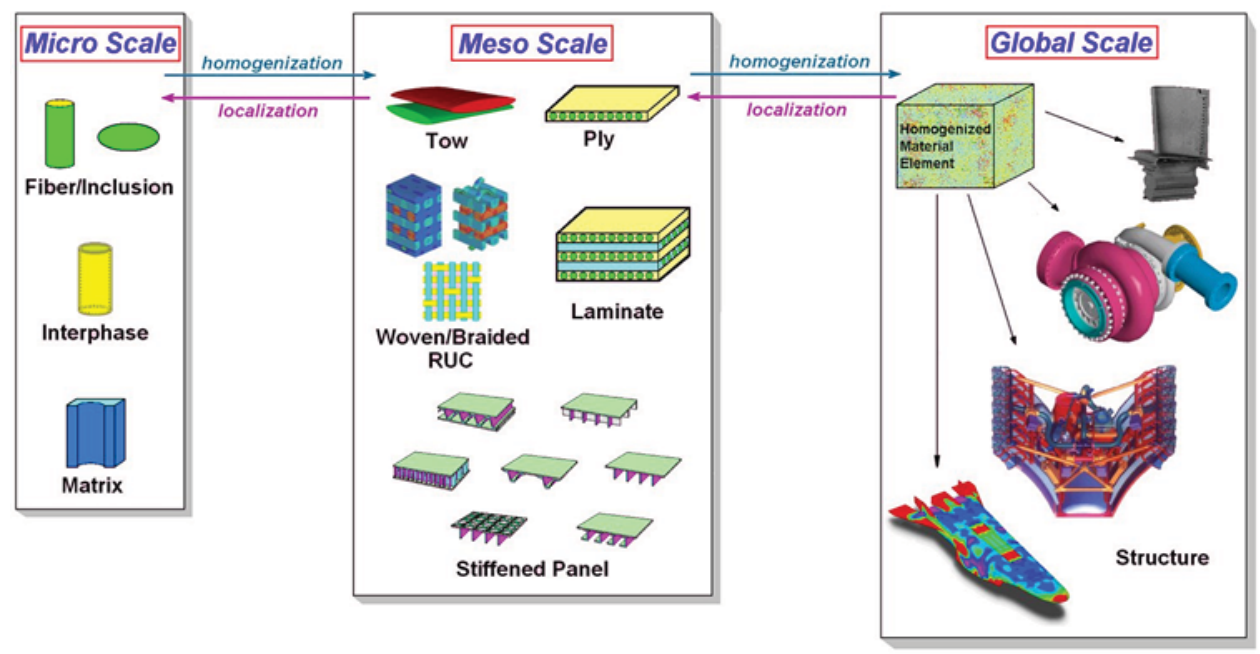

Figure 2: Illustration of relevant levels of scales for multiscale composite analysis.

One promising lamina-level theory for modeling matrix microdamage is Schapery theory (ST) ${ }^{2-7}$ a thermodynamically-based work potential model. ST was successfully used previously to accurately predict the behavior of numerous off-axis unidirectional, angle-ply and multi-angle laminates. ${ }^{4}$ However, lacking 
explicit microstructural details such as fiber volume fraction and fiber packing, this theory cannot be utlized to design the material (i.e., for ICME) since any such modification would constitute a "new material" and require re-characterization of the model. Therefore, the scope of its usage is limited to strictly analysis.

Micromechanics differs from macromechanics in that it enables one to account explicitly for variations in constituent material properties enhancements as well as microstructural architectural effects such as fiber volume content, fiber packing, void/defect content and orientations making micromechanics based models particularly attractive for use in ICME of composite materials. In this work, MAC/GMC, ${ }^{8,9}$ which uses the generalized method of cells (GMC) micromechanics theory, is presented as a computational tool suitable for achieving the required microstructure to property and property to performance relationships necessary to enable the application specific tailoring of the carbon fiber-reinforced, polymer matrix composites (PMCs).

Due to prior success in using ST, the current objective is to demonstrate that MAC/GMC can give comparable accuracy in predicting composite behavior while still enabling microstructural tailoring of the material. Since ST was formulated assuming, transversely isotropic lamina, it is not suitable for inclusion in a micromechanics model where the matrix phase requires a full $3-\mathrm{D}$, and often isotropic, formulation. Instead, a multi-axial mixed-mode continuum damage mode ${ }^{10}$ (MMCDM) is utilized to model the nonlinear behavior of the matrix, due to the accumulation of microdamage within a PMC repeating unit cell (RUC). This micromechanics model is calibrated to match the same experimental data, which was used to characterize the microdamage input functions for ST. Results from the calibrated micromechanics model, using MMCDM, are compared to the ST and experimental results for additional off-axis, angle ply, and multi-angle laminates.

Finally, manufacturing sensitive parameters such as fiber volume fraction and fiber packing are varied to observe the influence of these parameters on the ST input (microdamage function) and the performance of the multi-angle laminates. The analyses performed herein only considers matrix microdamage are invalid once meso-cracking (transverse cracking), fiber breakage, delamination or any other more severe damage mechanisms arise.

\section{The Generalized Method of Cells}

GMC, first developed in Ref. 11, offers a very computational efficient means of calculating the local fields within a composite RUC. It is completely general, and can admit any number of different phases with arbitrary deformation and damage constitutive laws, making it ideal for ICME applications. GMC has been utilized thoroughly for progressive damage and failure analysis (PDFA) of composite materials. ${ }^{10,12,13}$ As such, GMC is incredibly amenable for multiscale analysis of the same sort. ${ }^{7,14-16}$ A brief summary of the GMC fundamentals are presented in this section; the reader is referred to Ref. 1 for more details on GMC and the high-fidelity generalized method of cells (HFGMC).

With GMC, an RUC must be identified within the composite microstructure, and the RUC is discretized into a number of subcells, see Figure 3. Each subcell contains a single constituent (obeying the constitutive law of that constituent), but there is no limit on the number of different constituents that may be present in the RUC. Displacement and traction continuity is enforced in an average, or integral sense at each of the subcell interfaces and the periodic boundaries of the RUC. These continuity conditions are used to formulate a strain concentration matrix $\mathbf{A}$,

$$
\epsilon_{S}=\mathbf{A} \bar{\epsilon}
$$

which gives all the local subcell strains $\left(\boldsymbol{\epsilon}_{S}\right)$ in terms of the six, global, average, applied strains $\overline{\boldsymbol{\epsilon}}$.

Once the strain concentration matrix is formulated and the local subcell strains have been calculated, the local subcell stresses $\left(\boldsymbol{\sigma}^{(\alpha \beta \gamma)}\right)$ can be calculated using the local constitutive law and the local subcell strains.

$$
\boldsymbol{\sigma}^{(\alpha \beta \gamma)}=\mathbf{C}^{(\alpha \beta \gamma)} \boldsymbol{\epsilon}^{(\alpha \beta \gamma)}
$$

where $\alpha, \beta$, and $\gamma$ are the subcell indices in the local $y_{1^{-}}, y_{2^{-}}$, and $y_{3}$-directions, respectively, $\mathbf{C}^{(\alpha \beta \gamma)}$ is the local subcell stiffness and $\boldsymbol{\epsilon}^{(\alpha \beta \gamma)}$ contains the six local subcell strains. Eqs. (1) and (2) can be modified to admit inelasticity, however this manuscript focuses on stiffness reduction damage only. Finally, the global RUC stiffness can be calculated.

$$
\mathbf{B}=\frac{1}{D H L} \sum_{\alpha=1}^{N_{\alpha}} \sum_{\beta=1}^{N_{\beta}} \sum_{\gamma=1}^{N_{\gamma}} d_{\alpha} h_{\beta} l_{\gamma} \mathbf{C}^{(\alpha \beta \gamma)} \mathbf{A}^{(\alpha \beta \gamma)}
$$




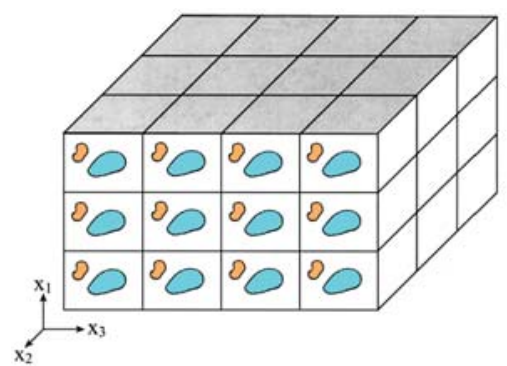

(a) Triply-periodic composite microstructure.

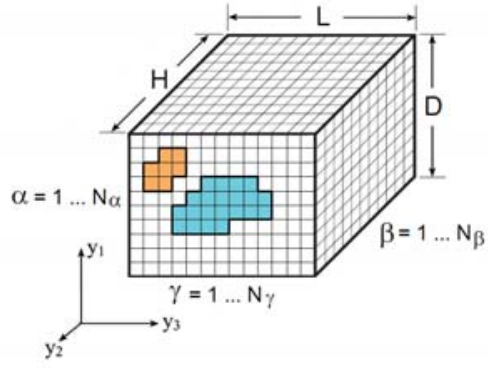

(b) Method of cells idealization of triply-periodic RUC.

Figure 3: Composite with repeating microstructure and arbitrary constituents.

where $N_{\alpha}, N_{\beta}$, and $N_{\gamma}$ are the total number of subcells in the $y_{1^{-}}, y_{2^{-}}$, and $y_{3}$-directions, respectively, $D$, $H$, and $L$ are the RUC dimensions in the $y_{1^{-}}, y_{2^{-}}$, and $y_{3}$-directions, $d_{\alpha}, h_{\beta}$, and $l_{\gamma}$ are the local subcell dimensions in the $y_{1^{-}}, y_{2^{-}}$, and $y_{3}$-directions for subcell $\alpha \beta \gamma$, and $\mathbf{A}^{(\alpha \beta \gamma)}$ is a submatrix of the strain concentration matrix A corresponding to subcell $\alpha \beta \gamma$.

\section{Multiaxial Mixed-mode Continuum Damage Model for Matrix Constituents}

\section{III.A. Damage Initiation}

The MMCDM, developed in Ref. 10 was implemented within MAC/GMC, and used to model progressive damage in the matrix constituent subcells. Damage initiation in each subcell is determined using quadratic definitions of damage strain $\epsilon_{1}^{D}, \epsilon_{2}^{D}, \epsilon_{3}^{D}$ (a 3-D extension of the strain-based Hashin criterion ${ }^{17}$ ).

$$
\begin{aligned}
& \epsilon_{1}^{D}=\sqrt{\left(\frac{\epsilon_{11}}{X_{\epsilon}}\right)^{2}+\left(\frac{\gamma_{13}}{R_{\epsilon}}\right)^{2}+\left(\frac{\gamma_{12}}{S_{\epsilon}}\right)^{2}} \\
& \epsilon_{2}^{D}=\sqrt{\left(\frac{\epsilon_{22}}{Y_{\epsilon}}\right)^{2}+\left(\frac{\gamma_{23}}{Q_{\epsilon}}\right)^{2}+\left(\frac{\gamma_{12}}{S_{\epsilon}}\right)^{2}} \\
& \epsilon_{3}^{D}=\sqrt{\left(\frac{\epsilon_{33}}{Z_{\epsilon}}\right)^{2}+\left(\frac{\gamma_{23}}{Q_{\epsilon}}\right)^{2}+\left(\frac{\gamma_{13}}{R_{\epsilon}}\right)^{2}}
\end{aligned}
$$

where $\epsilon_{i i}$ are the normal strain components, $\gamma_{i j}$ are the engineering shear strains, $Q_{\epsilon}, R_{\epsilon}$, and $S_{\epsilon}$ are the shear strain allowables, and the normal strain allowables $X_{\epsilon}, Y_{\epsilon}$, and $Z_{\epsilon}$ are given by:

$$
X_{\epsilon}=\left\{\begin{array}{cc}
X_{\epsilon}^{T} & \sigma_{11}>0 \\
X_{\epsilon}^{C} & \sigma_{11}<0
\end{array} ; \quad Y_{\epsilon}=\left\{\begin{array}{cc}
Y_{\epsilon}^{T} & \sigma_{22}>0 \\
Y_{\epsilon}^{C} & \sigma_{22}<0
\end{array} ; \quad Z_{\epsilon}=\left\{\begin{array}{cc}
Z_{\epsilon}^{T} & \sigma_{33}>0 \\
Z_{\epsilon}^{C} & \sigma_{33}<0
\end{array}\right.\right.\right.
$$

where $\sigma_{i i}$ are the normal stress components and the superscript ' $T$ ', or ' $C$ ', on the allowables indicates wether it is a compressive or tensile allowable. Damage initiates when any of the following inequalities are satisfied.

$$
\epsilon_{1}^{D} \geq 1 ; \quad \epsilon_{2}^{D} \geq 1 ; \epsilon_{3}^{D} \geq 1
$$

\section{III.B. Damage Evolution}

Once any of the inequalities in Eq. (6) is satisfied, damage begins to evolve in the matrix. It is assumed that the damage takes the form of an array of microcracks aligned perpendicular to the direction of the normal component of strain contributing to the damage strain (see Eq. (4)) that has exceed one. These microcracks grow perpendicular to $x_{i}$ under mixed-mode (I, II, III) loading conditions, as shown in Figure 4, where $\tau_{i j}$ are the shear stress parallel to the microcrack faces. 


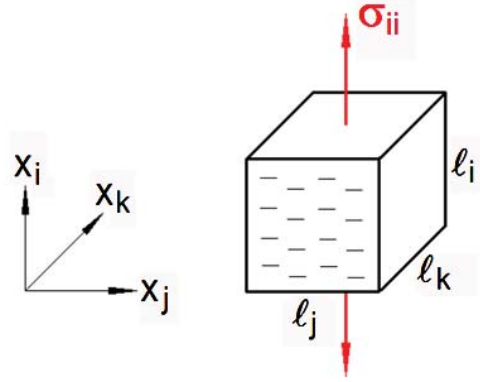

Mode I

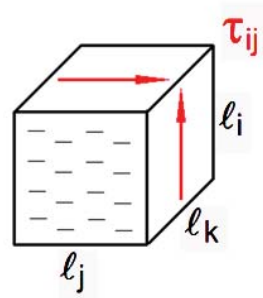

Mode II

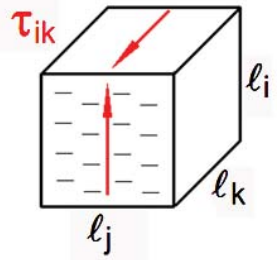

Mode III

Figure 4: Three loading modes that affect damage in the $x_{i}$ direction.

As such, damage is introduced through six scalar damage variables $D_{i}^{T}$ and $D_{i}^{C}$, where $i=1,2,3$ is determined from the damage $\operatorname{strain}(\mathrm{s}) \epsilon_{i}^{D}$ that exceeded one. It is assumed that the damage variables serve to degrade the engineering material properties.

$$
\begin{aligned}
E_{1}= & d_{1} E_{1}^{0} ; \quad E_{2}=d_{2} E_{2}^{0} ; \quad E_{3}=d_{3} E_{3}^{0} \\
& \nu_{12}=d_{1} \nu_{12}^{0} ; \quad \nu_{21}=d_{2} \nu_{21}^{0} ; \\
& \nu_{13}=d_{1} \nu_{13}^{0} ; \quad \nu_{31}=d_{3} \nu_{31}^{0} ; \\
& \nu_{23}=d_{2} \nu_{23}^{0} ; \quad \nu_{32}=d_{3} \nu_{32}^{0}
\end{aligned}
$$

where $E_{i}$ are the degraded Young's moduli, $E_{i}^{0}$ are the virgin Young's moduli, $\nu_{i j}$, and $\nu_{i j}^{0}$ are the degraded and virgin Poisson's ratios, respectively. The degradation factors, $d_{i}$ are calculated from the scalar damage variables.

$$
d_{i}=\left\{\begin{array}{cc}
1-b_{i i}^{T} D_{i}^{T} & \sigma_{i i}>0 \\
1-b_{i i}^{C} D_{i}^{C} & \sigma_{i i}<0
\end{array} \quad \text { no sum on } i, i=1,2,3\right.
$$

where $b_{i i}^{T}$ and $b_{i i}^{C}$ are parameters that allow the dependence of the moduli on the damage variables to be weighted differently in tension and compression. By default $b_{i i}^{T}=b_{i i}^{C}=1$. Note that, the Possion's ratios are degraded in Eq. (7) in such a manner that the off diagonal terms of the compliance matrix remain constant $S_{i j}=S_{i j}^{0},(i \neq j)$.

Microcrack evolution also affects the shear moduli.

$$
\begin{aligned}
G_{23} & =\left(1-b_{42} D_{2}^{\bullet}-b_{43} D_{3}^{\bullet}\right) G_{23}^{0} \\
G_{13} & =\left(1-b_{51} D_{1}^{\bullet}-b_{53} D_{3}^{\bullet}\right) G_{13}^{0} \\
G_{12} & =\left(1-b_{61} D_{1}^{\bullet}-b_{62} D_{2}^{\bullet}\right) G_{12}^{0}
\end{aligned}
$$

where

$$
D_{i}^{\bullet}=\left\{\begin{array}{cc}
D_{i}^{T} & \sigma_{i i}>0 \\
D_{i}^{C} & \sigma_{i i}<0
\end{array}\right.
$$

$G_{i j}$ are the degraded shear moduli, and $G_{i j}^{0}$ are the undamaged shear moduli. The shear moduli are affected by a linear, weighted combination of two corresponding damage variables. The contributing damage variables are associated with the two normals of shear stress components related to the shear modulus of interest. The default weighting factors $b_{i j}$ are equal to 0.5 .

Evolution of the damage variables $D_{i}$ is controlled by damage stress $\sigma_{i}^{D}$ versus damage strain $\epsilon_{i}^{D}$ constitutive laws, as shown in Figure 5. From this curve the damage increment can be determined.

$$
d D_{i}=\left(1-D_{i}-k_{i}^{\prime}\right) \frac{d \epsilon_{i}^{D}}{\epsilon_{i}^{D}}
$$

where $D_{i}$ is the damage state at the previous increment, $d \epsilon_{i}^{D}$ is the damage strain increment, $k_{i}^{\prime}=k_{i} / E_{i}^{0}$, and $k_{i}$ is the instantaneous tangent stiffness of the damage stress-damage strain curve. For full details on the derivation of Eq. (11), please refer to Ref. 10. In addition, Ref. 10 contains additional MMCDM theory 
used to control the failure of the material due to meso/maco cracking using the fracture toughness of the material. The focus of this current work is limited to microdamage, so that portion of the theory has been purposely omitted.

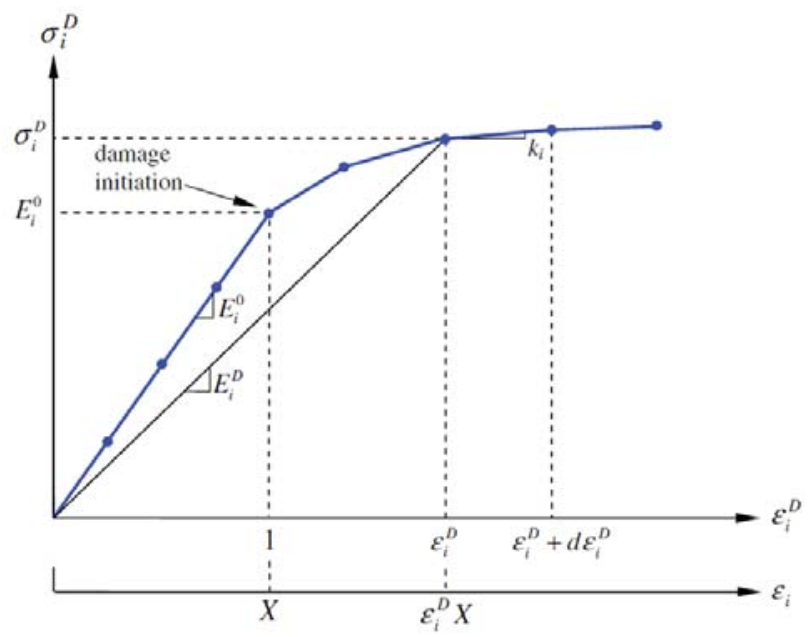

Figure 5: An incremental elastic damage stress versus damage strain curve. Note that $X=X_{\epsilon}, Y_{\epsilon}$, or $Z_{\epsilon}$.

\section{Schapery Theory for Microdamage Modeling at the Lamina Level}

ST is an anisotropic, thermodynamically-based, work potential theory for continuous fiber-reinforced laminae. ${ }^{2,3,5}$ It is capable of predicting the non-linear response of a composite lamina with evolving microdamage. When coupled with classical lamination theory, or the finite element method (FEM), ST can be used to predict the non-linear behavior of composite laminates, or structures. ${ }^{6,7,15,18}$ ST was used to model several different laminates in Ref. 4; the predictions were extremely accurate up to the onset of more catastrophic mechanisms such as meso/macro-matrix cracking, fiber breakage and/or delamination. ST has been since expanded to account for these other mechanisms. ${ }^{4,5,19-22}$ However, the scope of this work is limited to microdamage evolution, so the original formulation of ST is used.

ST assumes the total work potential $W_{T}$ can be partitioned into an elastic, recoverable portion $W$ and a dissipated portion $S$.

$$
W_{T}=W+S
$$

Figure 6 shows a representative, uniaxial, non-linear stress-strain curve. The recoverable, elastic strain energy density $W$ is shown as the area within the shaded triangle underneath a linear unloading line passing through the origin (ST assumes that there is no plastic deformation in the matrix, a reasonable assumptions for many $\mathrm{PMCs}^{23,24}$ ). The dissipated potential $S$ is the area under the stress-strain curve, above the unloading line. The internal state variable (ISV) $S$ is a measure of the amount of work potential used to facilitate structural changes in the material associated with microdamage. Microdamage is the nucleation and evolution of microcracks, shear bands, and fiber-matrix debonds in the matrix of the composite and is the primary mechanism for non-linearity in the stress strain response of a composite lamina up to the onset of more severe mechanisms such as transverse cracking, fiber breakage or delamination.

Invoking the stationarity of the total work potential with respect to the ISV, and defining the strain energy density for a composite lamina yields the ST evolution equation for the microdamage ISV.

$$
\frac{1}{2}\left(\epsilon_{22}^{2} \frac{d E_{22}}{d S}+\gamma_{12}^{2} \frac{d G_{12}}{d S}\right)=-1
$$

The only elastic properties that remain in Eq. (13) are $E_{22}$ and $G_{12}$ because the other properties, used to define $W$, are unaffected by matrix microdamage. In addition to Eq. (13), microdamage healing must be precluded.

$$
\dot{S} \geq 0
$$




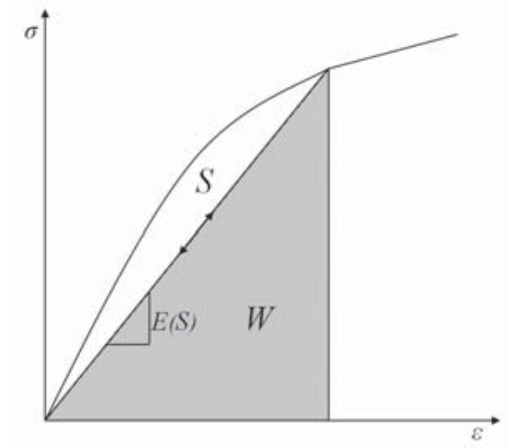

Figure 6: Non-linear stress strain curve showing the recoverable, elastic potential $W$, and the dissipated potential $S$.

For full details on the derivation of Eqs. (13)-(14), please see Refs. 6,7.

The damaged transverse and shear moduli $\left(E_{22}\right.$ and $\left.G_{12}\right)$ are related to their pristine values and the ISV through a set of microdamage functions $e_{S}^{T}, e_{S}^{C}$, and $g_{S}$.

$$
E_{22}=\left\{\begin{array}{ll}
E_{22}^{0} e_{S}^{T}(S) & \sigma_{22} \geq 0 \\
E_{22}^{0} e_{S}^{C}(S) & \sigma_{22}<0
\end{array} ; \quad G_{12}=G_{12}^{0} g_{S}(S)\right.
$$

The microdamage curves are measured from three uniaxial coupon tests. In Ref. 4 numerous off-axis unidirectional and angle-ply laminates were tested, and all laminates produced the same $g_{S}(S)$ and $e_{S}(S)$ $\left(e_{S}^{T}(S)\right.$ or $\left.e_{S}^{C}(S)\right)$ microdamage curves. The lay-up only affected the endpoint of the microdamage curve, which marks the transition to more severe damage and/or failure mechanisms. Eq. (15) can be used in Eq. (13) to solve for the ISV $S$ for a given strain state. In turn, Eq. (15) can then be applied to calculate the degraded moduli. Finally, the damage moduli can be used to calculate the corresponding stress state. Note that, ST does not require an initiation criterion. For low levels of strain, $S$ remains small, and due to the assumed microdamage functional form, does not affect the stiffnesses noticeably. However, as the strain levels increase, $S$ is amplified, and the non-linearity arises naturally.

The inputs to ST are the set of measured microdamage functions which relate the degradation in the engineering parameters to the ISV. The MMCDM (within GMC) was calibrated against the same experimental data used to obtain the ST inputs. In Section V.B the MMCDM is used to predict the stress-strain curves of the same laminates presented in Ref. 4 and compared to ST. The ultimate goal, presented in Section V.C, being to demonstrate the viability of GMC for ICME in a multiscale framework by varying the microstructure of the composite, at the lower scale, and observe its effects, at the higher scales, on the ST inputs and the performance of multi-angle laminates. The expectation is to achieve a similar level of accuracy to that of ST on the global scale while providing the ability to modify microstructural details at the constituent scale. Ideally, this scale linking could be extended further by tying the constitutive behavior of the matrix to a polymer processing model. Then, the inputs of GMC would be coming as outputs from other models and the constituent properties, composite microstructure and laminate lay-up could be tailored to meet application specific performance requirementes, thus achieving true ICME.

\section{Results}

\section{V.A. MMCDM calibration}

Experimental data and ST results from Ref. 4 were used to calibrate and validate the GMC model. The elastic lamina properties measured for AS4/3502 are given in Table 1, where $E_{11}$ is the modulus in the axial (fiber) direction, $E_{22}$ is the transverse modulus, $\nu_{12}$ is the Poisson's ratio, and $G_{12}$ is the shear modulus. These properties were used to back out the elastic properties of the fiber and matrix, given in Table 2, used in the 7x7, doubly-periodic, square pakced RUC (shown in Figure 7) which represents continuous reinforcement in the $y_{1}$-direction. The matrix (represented by green subcells in Figure 7) was assumed isotropic with a Young's modulus $E^{m}$ and a Poisson's ratio $\nu^{m}$, and the fiber (shown in Figure 7 with blue subcells) was assumed transversely isotropic, where $E_{11}^{f}$ is the longitudinal modulus, $E_{22}^{f}$ is the transverse modulus, $\nu_{12}^{f}$ 
is the axial Poisson's ratio, and $G_{12}^{f}$ is the axial shear modulus. Ref. 4 reported a volume fraction of $58 \%$. Note that even though the picture of the RUC in Figure 7 is 2-D, the subcell fields are full 3-D (they just do not vary in the $y_{1}$-direction); so, for a transversely isotropic material, the transverse Poisson's ratio $\nu_{23}^{f}$ is needed to fully characterize the stiffness tensor of the material. It was assumed that $\nu_{12}^{f}=\nu_{23}^{f}$.

Table 1: Elastic properties for AS4/3502 lamina. ${ }^{4}$

\begin{tabular}{|l|l|}
\hline Property & Value \\
\hline \hline$E_{11}$ & $125.8 \mathrm{GPa}$ \\
\hline$E_{22}$ & $9.31 \mathrm{GPa}$ \\
\hline$\nu_{12}$ & 0.329 \\
\hline$G_{12}$ & $5.10 \mathrm{GPa}$ \\
\hline
\end{tabular}

Table 2: Elastic properties for AS4 fiber and 3502 matrix constituents.

\begin{tabular}{|l|l|l|l|}
\hline Fiber Property & Value & Matrix Property & Value \\
\hline \hline$E_{11}^{f}$ & $214.3 \mathrm{GPa}$ & $E^{m}$ & $3.53 \mathrm{GPa}$ \\
\hline$E_{22}^{f}$ & $18.7 \mathrm{GPa}$ & $\nu^{m}$ & 0.35 \\
\hline$\nu_{12}^{f}$ & 0.32 & & \\
\hline$\nu_{23}^{f}$ & 0.32 & & \\
\hline$G_{12}^{f}$ & $81.4 \mathrm{GPa}$ & & \\
\hline
\end{tabular}

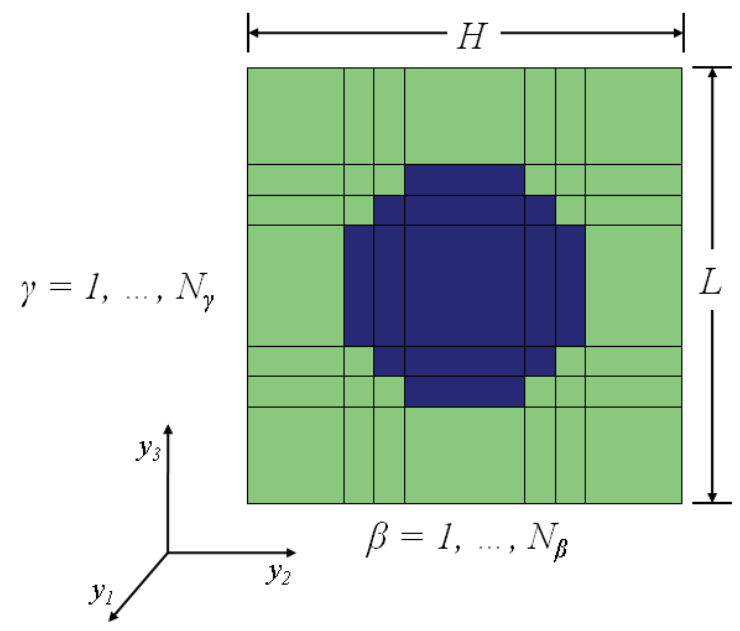

Figure 7: RUC used in square-packed analyses containing $7 \times 7$ subcell architecture. Blue subcells are composed of the AS4 fiber material, and green subcells are composed of 3502 matrix material. 
Data from three uniaxial coupon tests $\left(\left[ \pm 45^{\circ}\right]_{S},\left[15^{\circ}\right]\right.$, and $\left.\left[ \pm 30^{\circ}\right]_{S}\right)$ were used to characterize the three ST microdamage function inputs $\left(e_{S}\right), e_{S}^{C}(S)$, and $\left.g_{S}(S)\right)$ for AS4/3502. As mentioned before, many laminates were tested and produced the same microdamage curves. ${ }^{4}$ These three laminates provided the most data before the onset of a more catastrophic mechanism. The experimental data from the same three coupon tests were used to calibrate the inputs to the GMC micromechanics model using the MMCDM for matrix damage. Following Ref. 10, $k_{i}^{\prime}$ in Eq. (11) was taken to be an exponential function of the damage strain and two constants $A$ and $B$.

$$
k_{i}^{\prime}=A e^{\frac{-\epsilon_{i}^{D}}{B}}
$$

where

$$
A=\left\{\begin{array}{ll}
A^{T}, & \epsilon_{i}^{D}>0 \\
A^{C}, & \epsilon_{i}^{D}<0
\end{array} ; B= \begin{cases}B^{T}, & \epsilon_{i}^{D}>0 \\
B^{C}, & \epsilon_{i}^{D}<0\end{cases}\right.
$$

All of the parameters calibrated for the MMCDM are presented in Table 3.

Table 3: MMCDM parameters calibrated from $\left[ \pm 45^{\circ}\right]_{S},[15]$, and $\left[ \pm 30^{\circ}\right]_{S}$ experimental coupon data.

\begin{tabular}{|l|l|l|l|}
\hline Tensile Property & Value & Compressive Property & Value \\
\hline \hline$X_{\epsilon}^{T}$ & 0.0125 & $X_{\epsilon}^{C}$ & 0.0287 \\
\hline$S_{\epsilon}$ & 0.055 & - & - \\
\hline$A^{T}$ & 1.0 & $A^{C}$ & 1.0 \\
\hline$B^{T}$ & 1.0 & $B^{C}$ & 1.0 \\
\hline$b_{i i}^{T}$ & 0.75 & $b_{i i}^{C}$ & 0.1 \\
\hline$b_{4 i}, b_{5 i}, b_{6 i}$ & 0.5 & - & - \\
\hline
\end{tabular}

The resulting uniaxial stress strain curves for the $\left[ \pm 45^{\circ}\right]_{S},\left[15^{\circ}\right]$, and $\left[ \pm 30^{\circ}\right]_{S}$ laminates are presented in Figure 8. The MMCDM was able to correlate well for all three laminates. Some deviation can be observed at large strains in the $\left[15^{\circ}\right]$ and $\left[ \pm 45^{\circ}\right]_{S}$ shown in Figures 8a and 8c, respectively.

Figure 9 shows the microdamage curves measured from experiment ${ }^{4}$ (that where also used as input for characterizing ST), and the microdamage curves generated from the output of the micromechanics model used to calculate the stress-strain curves in Figure 8. The degradation in the moduli (value of microdamage function) are plotted against a reduced ISV $S_{r}=S^{1 / 3}$ in Figure 9 (see Figure 6 for the definition of $S$ ). The use of the reduced ISV allows for the microdamage curves to be fit with polynomial functions. The micromechanics results correlate best with to $e_{S}^{T}$ for the $\left[15^{\circ}\right]$ laminate, shown in Figure 9a.

The experimental microdamage curve for transverse (perpendicular to fiber) compression show an increase in moduli with increasing $S_{r}$, see Figure $9 \mathrm{~b}$. There are pre-existing micro-cracks, -voids, and -fissures in the composite prior to being loaded. Some irrecoverable energy is required to close these flaws, and as they are closed, the transverse stiffness of the plies increases. After the flaws are fully closed, further compression acts to slightly reduce the stiffness; yet, the effect is minimal. Since a multiplicative damage variable is utilized in MMCDM an increase in stiffness would require a negative damage increment. However, inadmissability of healing, in MMCDM, is enforced on the damage increment. ST also uses a multiplicative damage variable, but this variable is equal to a function, and inadmissability of healing is enforced on the ISV argument of that function. Thus, ST can admit a positive change in stiffness with a positive change in the dissipated potential. To admit an increase in the transverse stiffness with MMCDM, the "no healing" restriction imposed on the damage increment would need to be relaxed. This is physical incorrect though because damage is still increasing (i.e. energy is dissipated to advance structural changes in the material); under transverse compressive loading, the dissipated potential serves to advance changes that manifest as an increase in the observed stiffness. The discrepancy, between MMCDM and the experiment, in the transverse behavior of the plies had little effect however on the stress-strain response of the $\left[ \pm 30^{\circ}\right]_{S}$ laminate, as seen in Figure 8b.

The shear microdamage curve obtained from MMCDM deviates from the experimental curve for low values of $S_{r}$ (Figure 9c). This is because MMCDM require an initiation criterion, and $G_{12} / G_{12}^{0}$ will remain one until initiation criterion is satisfied. In the experiment, initial, low levels of dissipated potential act to degrade the shear modulus; however the degradation is small and does not significantly impact the stressstrain response of the $\left[ \pm 45^{\circ}\right]_{S}$. As $S_{r}$ increases the deterioration of $G_{12}$ is more apparent. ST can capture 


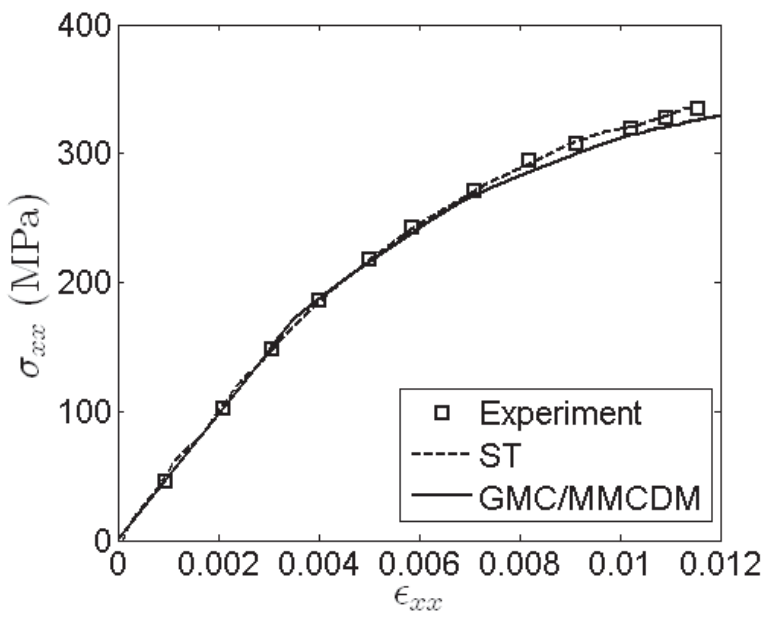

(a) $\left[15^{\circ}\right]$.

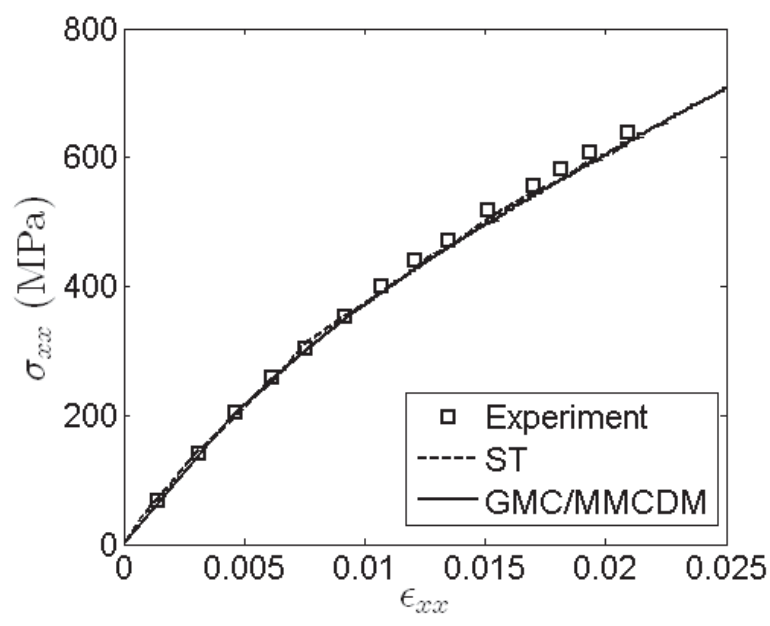

(b) $\left[ \pm 30^{\circ}\right]_{S}$.

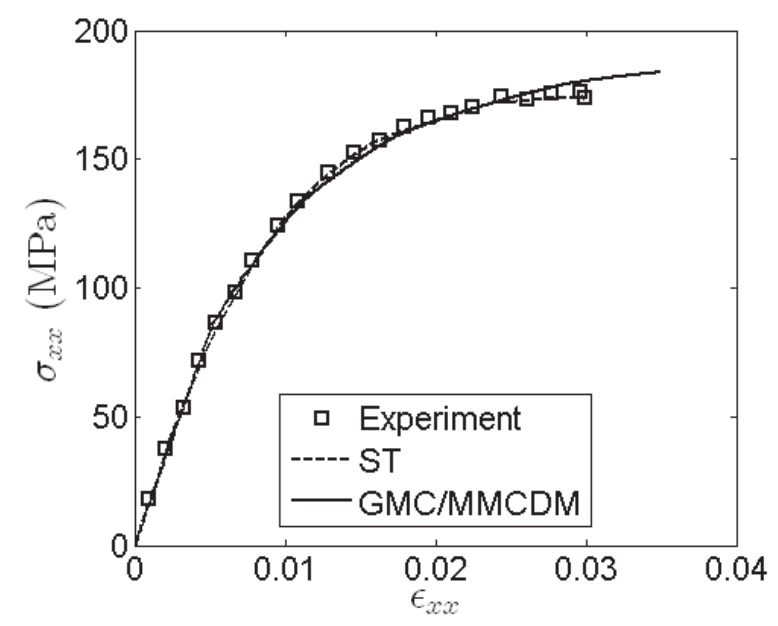

(c) $\left[ \pm 45^{\circ}\right]_{S}$.

Figure 8: Uniaxial stress-strain curves used to calibrate MMCDM and characterize ST. 


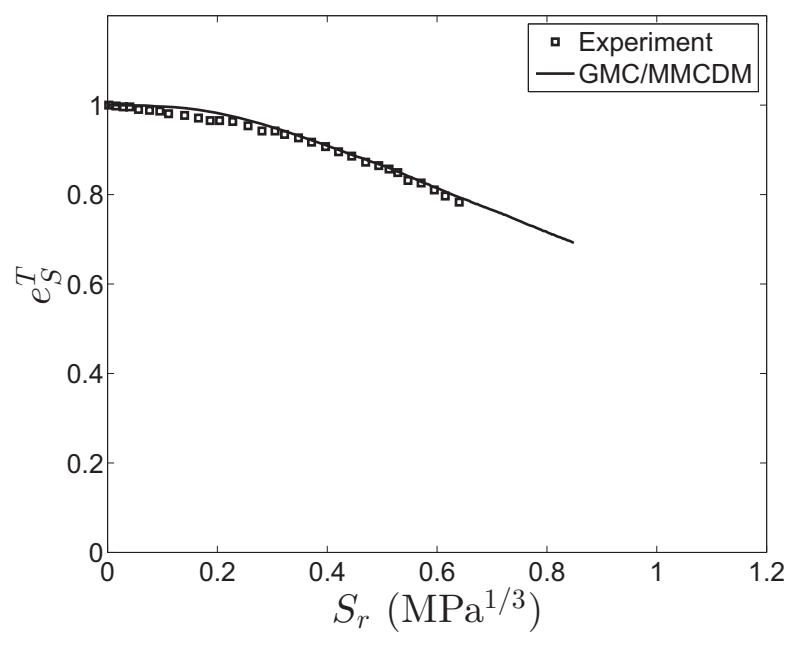

(a) Transverse Tension.

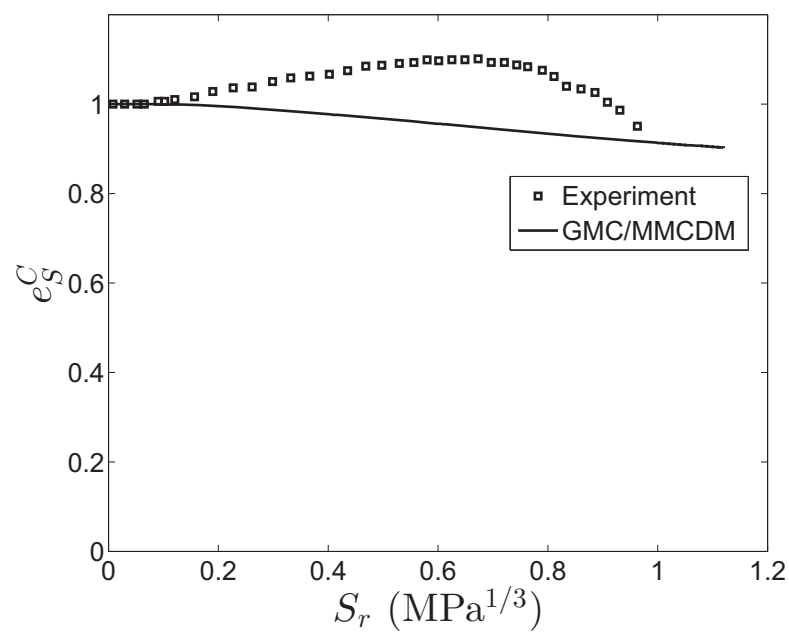

(b) Transverse Compression.

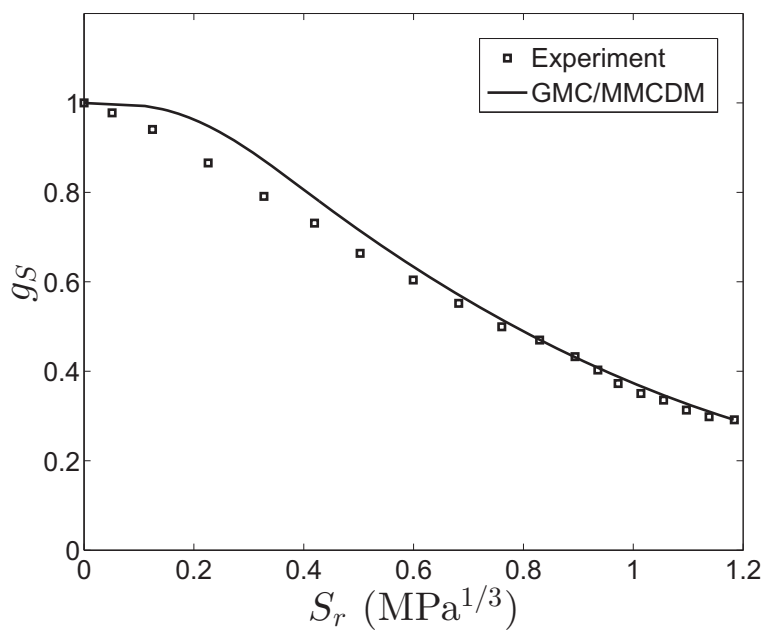

(c) Shear.

Figure 9: Matrix microdamage curves from experiment and GMC using MMCDM. 
this behavior directly because it does not require a failure criterion. Although there is a noticeable difference in the shear microdamage curve from MMCDM, compared to experiment, it is not evident in the stress-strain curves shown in Figure 8c. However, this indicates that the functional form of the damage evolution in the MMCDM, Eq. (11) may not be correct. If Eq. (11) utilized based on work potential, rather than strain, perhaps the predictions of the curves in Figure 9 using MMCDM would be improved

\section{V.B. MMCDM Predictions}

The calibrated properties, in Tables 2 and 3, were used in the MMCDM within GMC to predict the response of several different off-axis unidirectional and angle-ply laminates. The resulting stress versus strain curves are compared to experimental results from Ref. 4 in Figure 10. The focus of this work was to evaluate the capability of the MMCDM to predict the onset and evolution of matrix microdamage with the intent of subsequently simulating changes in the composite processing and manufacturing to improve the performance of the material, with respect to microdamage. Many of the experiments used to produce results presented in Figure 10 exhibited damage mechanisms beyond matrix microdamage, including transverse cracking and delamination. ST was validated as an accurate tool for predicting microdamage in PMC laminates, ${ }^{4}$ but deviated from the experimental results upon the onset of other damage mechanisms. Thus, the ST predictions, taken from Ref., ${ }^{4}$ are also included in Figure 10 to give a better indication of the ability of the MMCDM to predict microdamage. However, MMCDM is not restricted to modeling microdamage alone, and can be used to model the combining effects of all damage mechanisms. Whereas, ST would require a reformulation to capture the effects of other mechanisms.

The GMC model utilizing MMCDM is able to predict the non-linear response of simple laminates exhibiting matrix microdamage reasonably. Figures 10a-10c show some deviation from the ST predictions and experimental results at high levels of strain, especially in the $\left[45^{\circ}\right]$ and $\left[60^{\circ}\right]$ laminates. For the angle-ply laminates (Figures 10d - 10g), the GMC/MMCDM model was able to predict the non-linear behavior of the $\left[ \pm 15^{\circ}\right]_{S},\left[ \pm 50^{\circ}\right]_{S}$, and $\left[ \pm 60^{\circ}\right]_{S}$ laminates well, but there is significant error in the $\left[ \pm 40^{\circ}\right]_{S}$ laminate for strains greater than $2.5 \%$. The $\left[ \pm 40^{\circ}\right]_{S}$ laminate was loaded to four times the strain level of the other angle-ply laminates. As such, the disparity observed at the end of the $\left[ \pm 45^{\circ}\right]_{S}$, at approximately $3 \%$ strain, in Figure 8c continues to amplify with increasing strain in the $\left[ \pm 40^{\circ}\right]_{S}$ example. This suggests that a different functional form may need to be introduced into the MMCDM to more adequately capture the damage behavior.

Figure 11 shows the lamina microdamage curves predicted from the simulation of the same laminates, used to generate the stress-strain curves in Figure 10, with GMC utilizing the MMCDM. These microdamage curves are input for the ST macroscopic damage model, and as inputs, do not change based upon lay-up. Ref. 4 reported that these microdamage curves were also insensitive to changes in the lay-up experimentally, with the exception of the endpoint of the microdamage curves which marks the initiation of some other damage or failure mechanism. Thus, only a single curve is presented for each microdamage function, representing the experimental data.

The transverse tensile microdamage functions in Figure 11a predicted using GMC with MMCDM exhibit a fair degree of scatter. However, this did not directly correlate to poor accuracy when predicting the stress-strain curves, shown in Figure 10. The transverse compression microdamage curves are more or less coincident, but there is a an appreciable difference between the predicted curves and the experimental data for reasons explained previously. The predicted shear microdamage curves also exhibit accordance with each other in Figure 11b.

The laminates were loaded, in strain control, to the maximum strain reported in Ref. 4. At this point the laminates either failed or exhibited some other catastrophic damage mechanism. The endpoints of the predicted microdamage curves are marked with red symbols in Figure 11 and demonstrate that the transition to failure, or other damage mechanisms, is dependent on the lay-up and does not occur at a fixed value of $S_{r}$. This means that the driving force for microdamage is independent of the lay-up, but the driving force for other mechanisms is not.

Figure 12 shows the stress-strain curve predictions for three multi-angle laminates: $\left[ \pm 45^{\circ} / \pm 45^{\circ} / 90^{\circ} / \pm\right.$ $\left.45^{\circ}\right]_{S},\left[ \pm 45^{\circ} / 90_{2}^{\circ} / \pm 45^{\circ} / 90_{2}^{\circ} / \pm 45^{\circ}\right]_{S}$, and $\left[ \pm 45^{\circ} / 60_{2}^{\circ} / \pm 45^{\circ} / 60_{2}^{\circ} / \pm 45^{\circ}\right]_{S}$. Reasonable predictions were obtained with MMCDM for all three laminates, and these predictions match those for ST. Ref. ${ }^{4}$ reported the initiation of transverse cracks at axial strains of 0.004-0.005; however, the ST results do not deviate noticeably from the experiment until larger strains are achieved. This indicates that, initially, the effects of a small number of transverse cracks on the laminate stiffness is minimal but becomes more prominent as the 


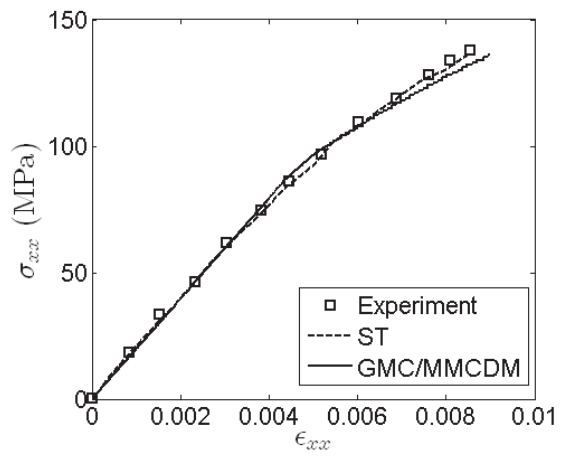

(a) $\left[30^{\circ}\right]$

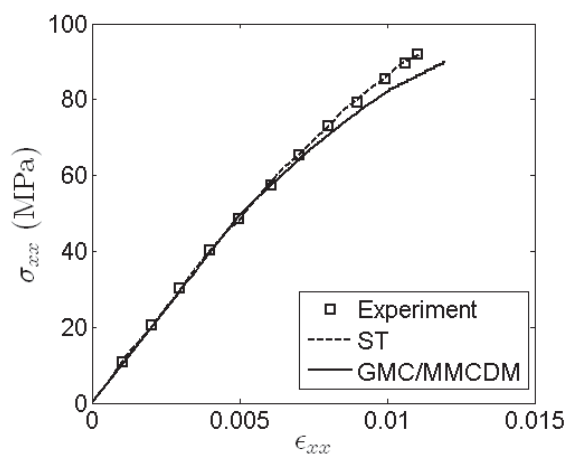

(c) $\left[60^{\circ}\right]$.

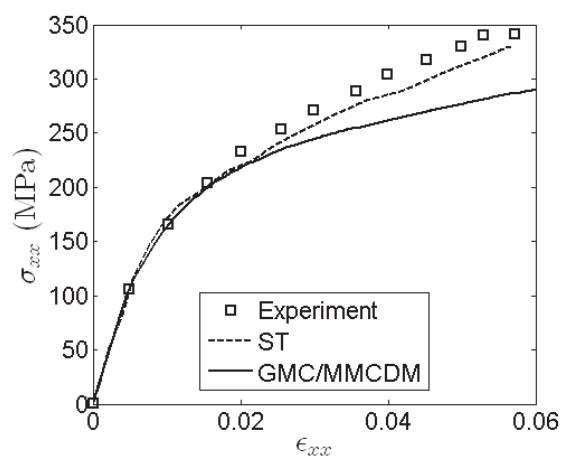

(e) $\left[ \pm 40^{\circ}\right]_{S}$.

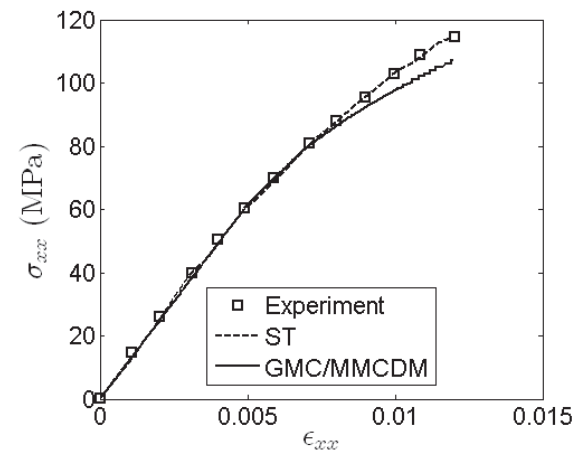

(b) $\left[45^{\circ}\right]$.

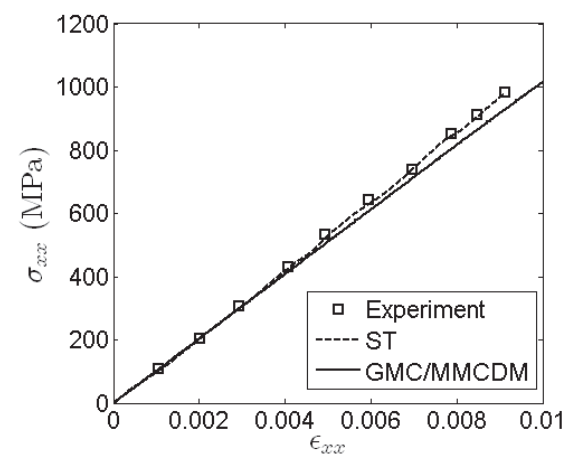

(d) $\left[ \pm 15^{\circ}\right]_{S}$.

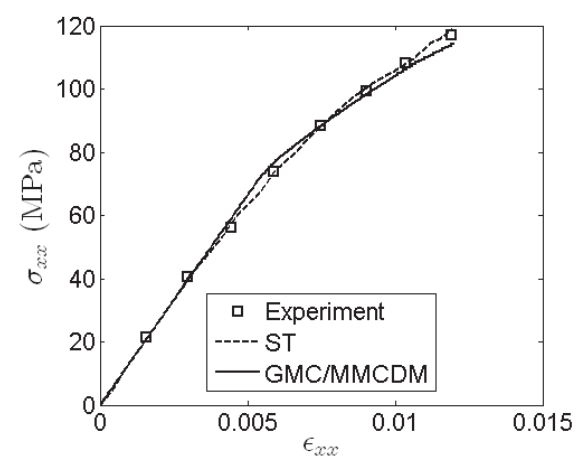

(f) $\left[ \pm 50^{\circ}\right]_{S}$.

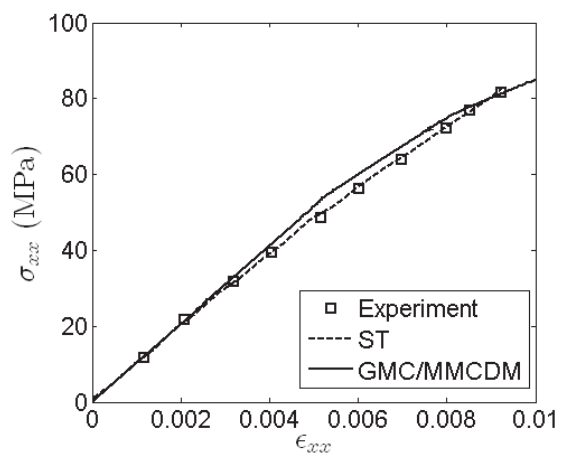

(g) $\left[ \pm 60^{\circ}\right]_{S}$.

Figure 10: Stress-strain curves predictions from GMC with MMCDM compared to experimental results ${ }^{4}$ for a variety of off-axis unidirectional and angle-ply laminates. 


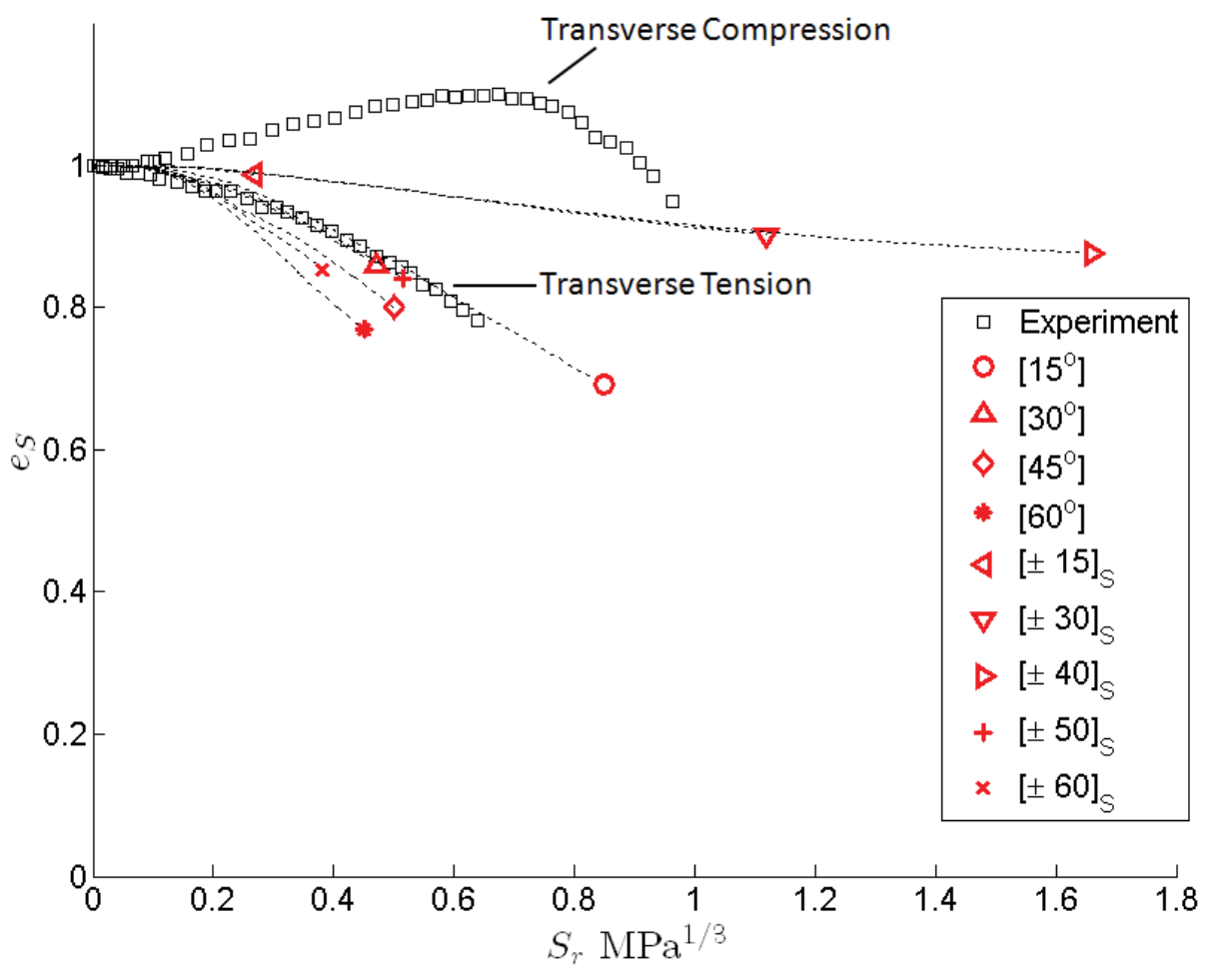

(a) Transverse.

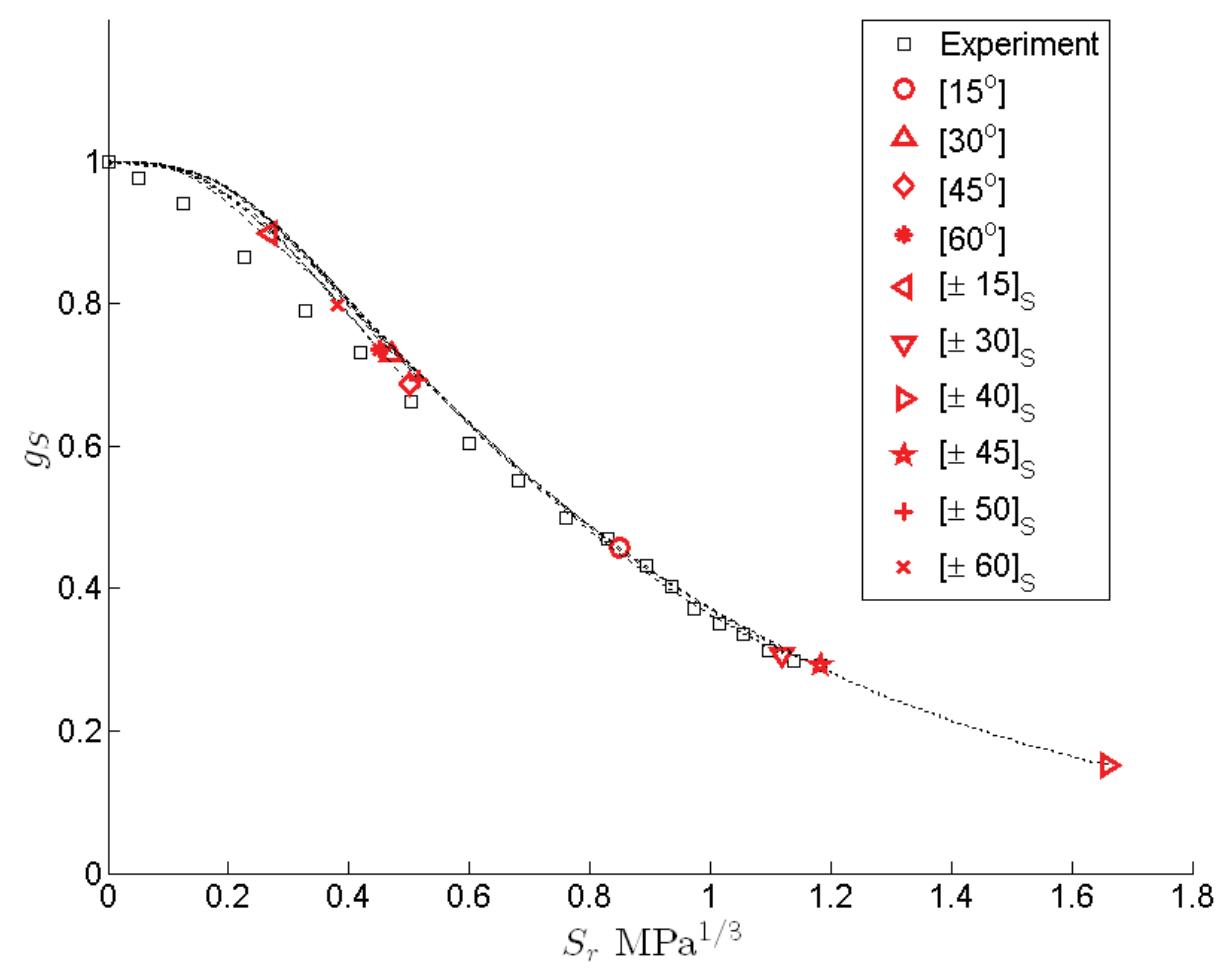

(b) Shear.

Figure 11: Matrix microdamage curves from GMC using MMCDM for a variety of laminates. 
crack multiply.

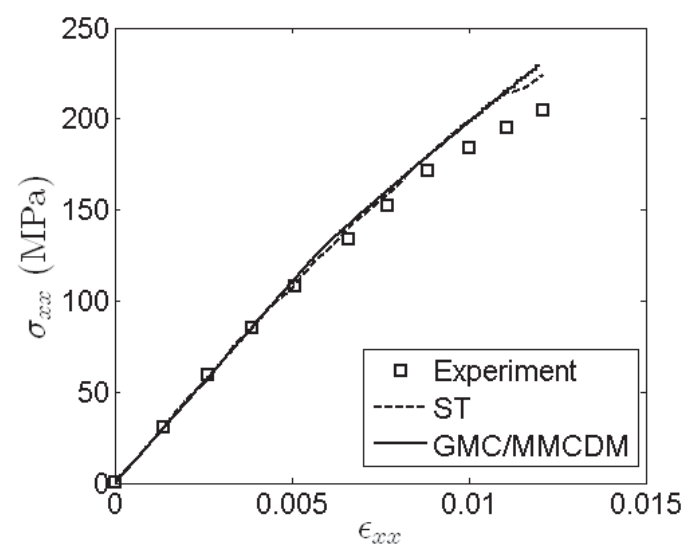

(a) $\left[ \pm 45^{\circ} / \pm 45^{\circ} / 90^{\circ} / \pm 45^{\circ}\right]_{S}$.

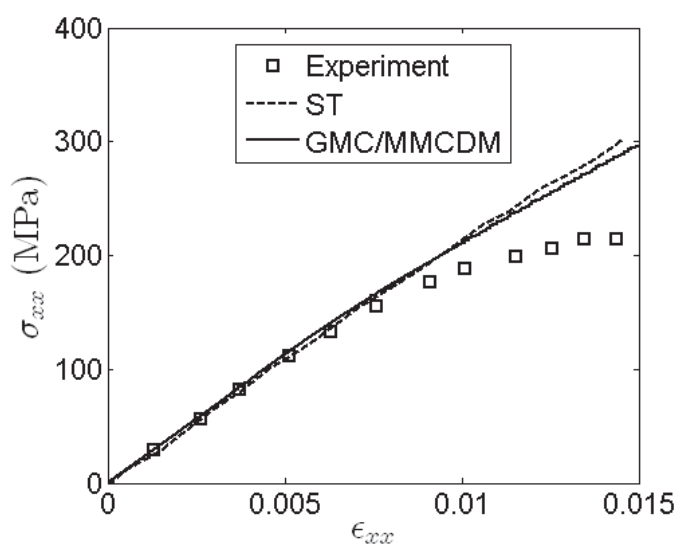

(b) $\left[ \pm 45^{\circ} / 90_{2}^{\circ} / \pm 45^{\circ} / 90_{2}^{\circ} / \pm 45^{\circ}\right]_{S}$.

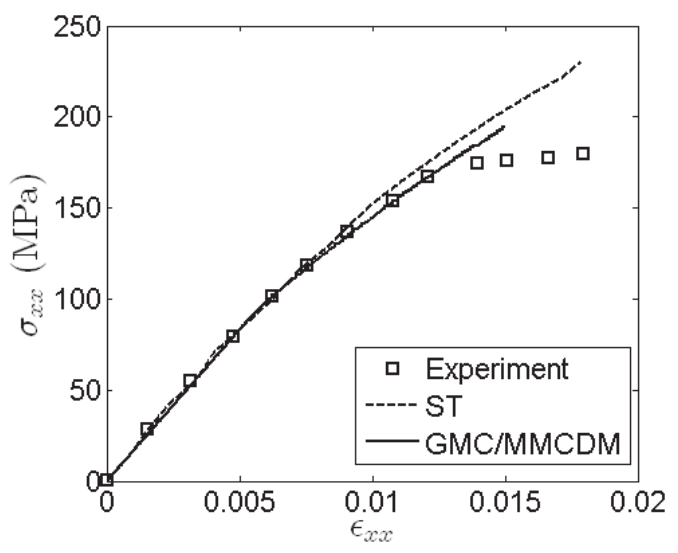

(c) $\left[ \pm 45^{\circ} / 60_{2}^{\circ} / \pm 45^{\circ} / 60_{2}^{\circ} / \pm 45^{\circ}\right]_{S}$.

Figure 12: Stress-strain curves predictions from GMC with MMCDM compared to experimental results ${ }^{4}$ for a three multi-angle laminates.

Figure 11 clearly indicates that the current damage model used to represent the matrix damage within GMC does not produce a commensurate amount of change in global stiffness with respect to the ISV $S$ (measure of dissipated work), nor are the predicted microdamage curves insensitive to laminate lay-up, as observed in the experiments. However, ST is able to predict the stress-strain behavior of composites experiencing matrix microdamage because the ISV chosen yields the correct driving thermodynamic driving force $f$ for microdamage evolution. The thermodynamic driving force is given by the change in the elastic strain energy density with resect to incremental changes in the ISV. ${ }^{3}$

$$
f=-\frac{d W}{d S}
$$

If $S$ was chosen inappropriately, then the thermodynamic driving force in the model would be incorrect. In addition, the functional forms of the degradation of $E_{22}$ and $G_{12}$ were also chosen correctly (measured directly from experiment), and moreover, are insensitive to the lay-up of the laminate.

The MMCDM is not a work potential theory, and accordingly does not utilize a thermodynamic force to control the evolution of microdamage. Yet, the parameters can be tuned to mimic the characterization experiments for ST and predict the response of other laminates within a fair degree of accuracy. Unfortunately, this makes it difficult to assess wether it is the constitutive model, the micromechanics, or both that are responsible for the observed discrepancies. To reduce potential error induced by the constitutive model a 3 -D work potential theory for initially isotropic materials ${ }^{15}$ will be used in the future to model the matrix. 
Confidence in the constitutive law could be achieved if the predicted microdamage curves were insensitive to the laminate lay-up, as observed in the experiments. It should also be mentioned that the flexibility of the MMCDM allows multiple mechanisms to be captured (although not demonstrated in this work), whereas, ST would require the addition of another ISV ${ }^{4,5,19}$ significantly increasing the complexity and computational cost of the theory. Moreover, carefully planned experiments must be devised to isolate and/amplify the effects of the mechanism of interest, relative to other mechanisms, and it must be determined that the choice of ISV for the new mechanism is correct and independent of lay-up and loading.

\section{V.C. Using GMC and MMCDM to Predict Processing Effects}

Although using the MMCDM within GMC was not able to identically reproduce the macroscopic, ST results, it can still be employed to interrogate the effects of microstructures which is linked with processing, such as fiber volume fraction and fiber packing, on the microdamage evolution in the matrix phase. Thus, demonstrating the potential usefullness for GMC within ICME applications. This type of study is not possible with $\mathrm{ST}$, or other macroscopic theories.

The fiber volume fraction of the $\left[15^{\circ}\right],\left[ \pm 30^{\circ}\right]_{S},\left[ \pm 45^{\circ}\right]_{S}$ were varied from $10 \%$ to $78.5 \%$ assuming the square packed architecture shown in Figure 7 to determine the effects of fiber volume fraction on the ST microdamage functions.

Figure 13 shows the normalized degradation in the appropriate moduli versus fiber volume fraction $V_{f}$, for a fixed damage state $\left(S_{r}=0.5\right)$, obtained from simulations of the three coupons. The transverse compression response is completely insensitive to changes in $V_{f}$. Under transverse tension the degradation of $E_{22}$ increased by $5 \%$ as $V_{f}$ increased. However, the damage began to lessen as $V_{f}$ goes above $60 \%$. The shear response was extremely sensitive to the fiber volume fraction and the damaged shear modulus steadily declined with an increase in $V_{f}$. After $V_{f}=50 \%$ the rate of degradation with respect to increasing $V_{f}$ became very rapid. According to Figure 13, a lower fiber volume fraction will lessen the severity of any resulting damage on shear modulus of a ply. Although, there will be a penalty in stiffness, the fixed damage state $\left(S_{r}=0.5\right)$ may be achieved at lower loads; therefore, there must be a balance.

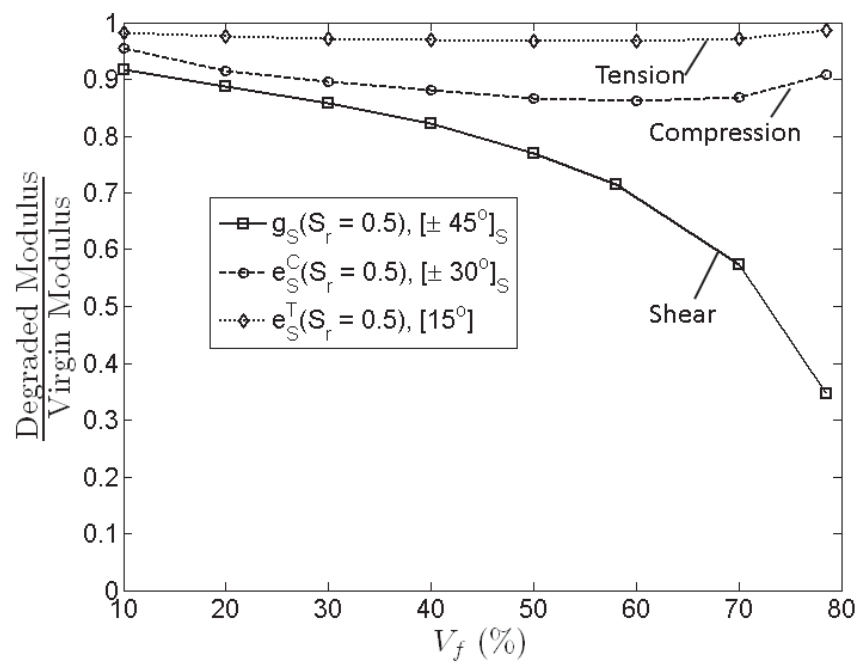

Figure 13: Effect of fiber volume fraction on modulus degradation for a fixed damage state $\left(S_{r}=0.5\right)$.

Since $V_{f}$ affects the stiffness of the laminates, comparing stress-strain curves does not accentuate the effects of fiber volume fraction on the microdamage evolution in the composite. Therefore, Figure 14 shows the fraction of the total work that is dissipated due to microdamage, for a fixed total work potential $W_{T}=2.5$ $\mathrm{MPa}$, as a function of fiber volume fraction, for the three characterization laminates and the three multiangle laminates. Most of laminates exhibited an increase in dissipated potential with fiber volume fraction. The amount of potential that was dissipated is more sensitive to changes in the $V_{f}$ for the $\left[ \pm 30^{\circ}\right]_{S}$ and $\left[ \pm 45^{\circ}\right]_{S}$ angle-ply laminates. The second multi-angle laminate, which had $40 \% 90^{\circ}$ plies, actually showed slight decrease in dissipated potential up to $40 \% V_{f}$ followed by an increase in $S / W_{T}$. Figure 14 shows that a lay-up and volume fraction can be chosen to minimize the fraction of the total, applied work potential that 
is used to facilitate microdamage evolution, but again, attention must be paid to stiffness and load carrying requirements of the structure.

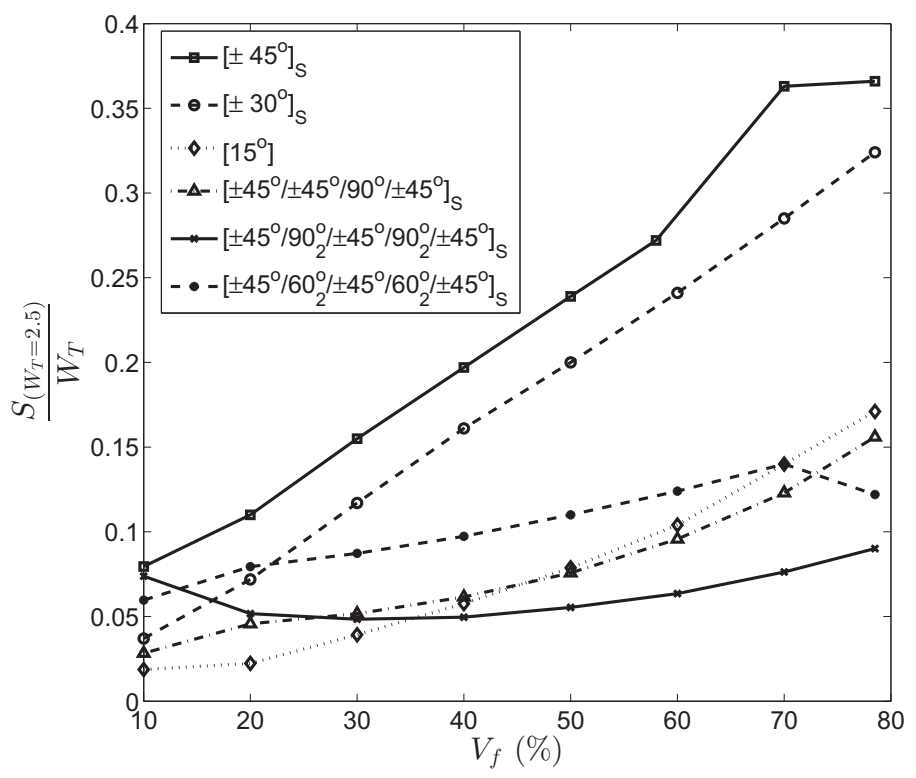

Figure 14: Fraction of total work potential that is dissipated versus fiber volume fraction for a fixed total work potential $W_{T}=2.5 \mathrm{MPa}$.

A preliminary investigation on the effect of packing on the performance of the material was conducted by changing the fiber architecture from a square packing (Figure 7) to a hexagonal packing, shown in Figure 15. The volume fraction was retained at $58 \%$, as well as the fiber geometry.

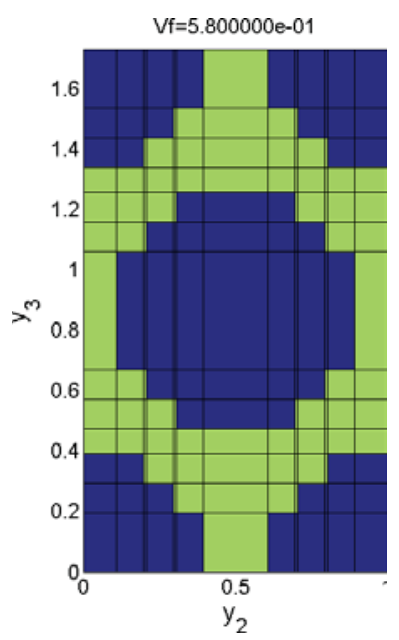

Figure 15: Hexagonally-packed fiber architecture.

Figure 16 shows the predicted microdamage curves for the two different microstructures using the characterization laminates $\left(\left[15^{\circ}\right],\left[ \pm 30^{\circ}\right]_{S},\left[ \pm 45^{\circ}\right]_{S}\right)$. The transverse tension and shear microdamage functions were only moderately sensitive to changes in the packing, but the transverse compression microdamage function was significantly affected by the change. However, it cannot be stated conclusively that $e_{S}^{C}$ is sensitive to the fiber packing because a good correlation for $e_{S}^{C}$ was not obtained for the baseline, square-packed architecture.

The stress-strain curves for the three multi-angle laminates predicted using MMCDM within GMC utilizing square and hexagonal packing architectures are displayed in Figure 17. The packing appears to have a minimal effect on the results for the multi-angle laminates. However, based on the results presented in Figure 16, the difference is expected to be more pronounced if the laminates are loaded in compression. 


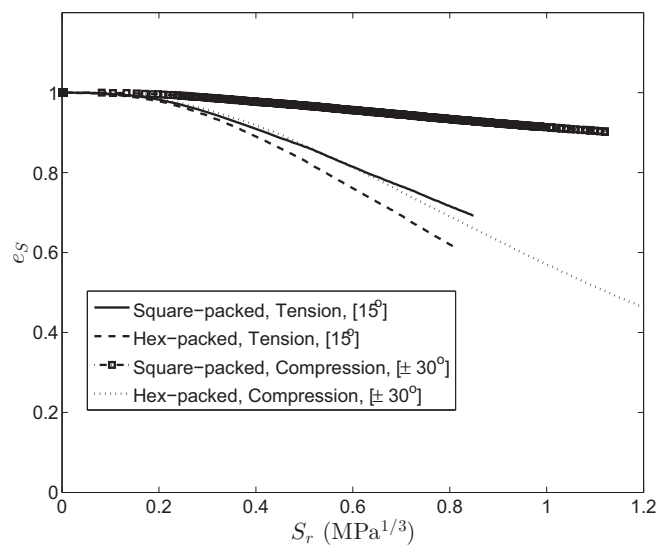

(a) Transverse.

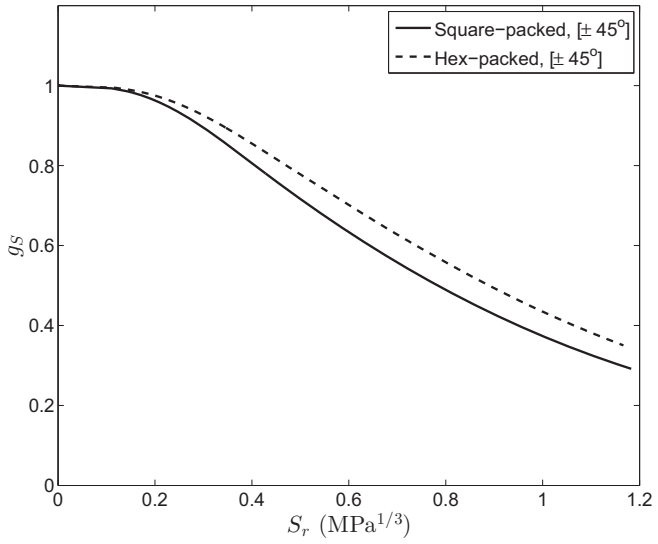

(b) Shear.

Figure 16: Matrix microdamage curves from GMC using MMCDM for two different fiber packing architectures.

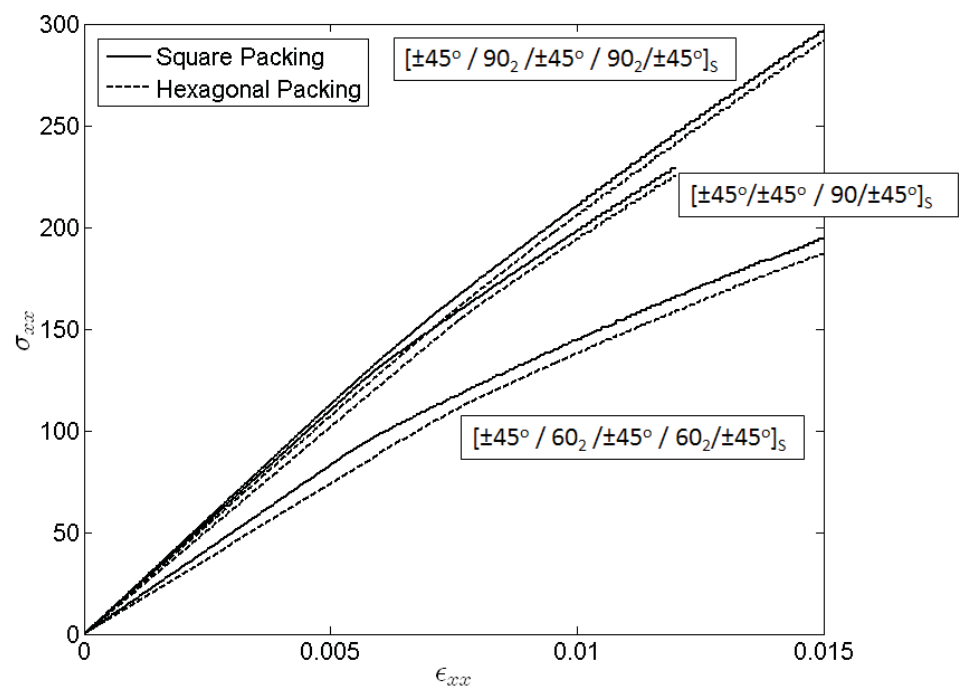

Figure 17: Stress-strain response of three multi-angle laminates for square-packed and hexagonally-packed architectures. 
It is difficult to make any definitive statements on the effects of fiber packing on the microdamage in the composite with only two cases. To make any solid conclusions the statistical repeating volume element (RVE) containing randomly packed would first need to be identified and used as a baseline for comparison with other perturbations of this system (the extreme cases being ordered microstructures such as square and hexagonally packed), as having the correct statistical RVE is important with respect to not only accurately concluding about packing effects but also whether or not one has the correct damage model. Moreover, the need to have accurate constitutive models are imperative, more so with micromechanics than at the macroscale since the in-situ fields are highly multiaxial and nonproportional.

\section{Conclusion}

The MMCDM was implemented within the MAC/GMC software suite and used to predict the response of numerous off-axis unidirectional, angle-ply, and multi-angle laminates exhibiting matrix microdamage. The matrix damage parameters used in the model were calibrated to match the response of three different coupon tests. These same three coupons were used in Ref. 4 to characterize the microdamage functions used in macroscopic ST damage model. The stress-strain curves predicted using MMCDM were compared to the experimental data and ST results. In addition, the microdamage functions predicted by MMCDM were compared to experiment.

Figures 8 - 12 demonstrate that the MMCDM, within a micromechanics theory, can correlate very well to data used to characterize the ST macromechanics model. However, the MMCDM did perform poorly in predicting the response of some laminates and exhibited a marginal amount of error when reproducing the matrix microdamage ST input functions. The discrepancy between ST and MMCDM was less apparent in multi-angle laminates. Ideally, an work potential-based damage theory, similar to ST, should be introduced into MAC/GMC to model the behavior of the matrix constituent.

ST offered accurate predictions for the response of laminates experiencing matrix microdamage evolution making it an effective constitutive model for use in FEM modeling of structures. However, the influence of changes in the material processing or microstructure cannot be determined with ST. With micromechanics (GMC), and MMCDM, parameters such as volume fraction and fiber packing can be traded and their effects on the damage evolution of the system can be observed. Furthermore, the micromechanics can be utilized to predict the ST microdamage functions, accounting for processing changes.

It was seen that changes in the volume fraction significantly affected the shear microdamage function. In addition, the fraction of total work potential that was dissipated due to microdamage (for a given applied total work potential) was sensitive to changes in the volume fraction for particular laminates, but insensitive to others. Finally, it seemed that changing the fiber packing architecture from square to hexagonal has a marked impact on the resulting transverse compression microdamage function. Further studies can be conducted to determine the effects of random architectures on the response of the laminates, but this will require a thorough statistical investigation to identify the statistical RVE. It is anticipated that the damage curves produced using a statistical RVE, within a laminate, will not be sensitive to the lay-up of the laminates because the statistical RVE is energetically objective when damage is present. ${ }^{25,26}$

Through these simulations, it has been demonstrated that GMC can be used as a viable tool for conducting ICME on composite materials while achieving comparable macrolevel results to an accurate macrolevel theory ST which lacks the ability to design the material. However, these are preliminary steps towards ICME, but to achieve true ICME the laminate stiffness, load-carrying capability, and susceptibility to microdamage must be tailored by varying the fiber volume fraction, microstructure, laminate lay-up, and material properties to meet some structural performance. Additionally, the behavior of the constituents should be tied to more fundamental materials science models to improve the robustness of the method and its applicability

to ICME. Moreover, the properties that are determined suitable to meet the required performance must be tied to material manufacturing and processing simulations or data to determine if they are achievable. 


\section{References}

${ }^{1}$ Aboudi, J., Arnold, S. M., and Bednarcyk, B. A., Micromechanics of Composite Materials: A Generalized Multiscale Analysis Approach, Elsevier, Inc., 2013.

${ }^{2}$ Schapery, R. A., "Mechanical characterization and analysis of inelastic composite laminates with growing damage," Mechanics \& Materials Center Report 5762-89-10, Texas A \& M University, College Station, TX 77804, 1989.

${ }^{3}$ Schapery, R. A., "A theory of mechanical behaviour of elastic media with growing damage and other changes in structure," J. Mech. Phys. Solids, Vol. 38, No. 2, 1990, pp. 1725-1797.

${ }^{4}$ Sicking, D. L., Mechanical Characterization of Nonlinear Laminated Composites with Transverse Crack Growth, Ph.D. thesis, Texas A\&M University, College Station, TX, 1992.

${ }^{5}$ Schapery, R. A. and Sicking, D. L., "A theory of mechanical behaviour of elastic media with growing damage and other changes in structure," Mechanical Behaviour of Materials, edited by A. Bakker, Delft University Press, Delft, The Netherlands, 1995, pp. 45-76.

${ }^{6}$ Basu, S., Waas, A. M., and Ambur, D. R., "Prediction of progressive failure in multidirectional composite laminated panels," Int. J. Solids Structures, Vol. 44, No. 9, 2007, pp. 2648-2676.

${ }^{7}$ Pineda, E. J., Waas, A. M., Bednarcyk, B. A., Collier, C. S., and Yarrington, P. W., "Progressive damage and failure modeling in notched laminated fiber reinforced composites," Int. J. Fract., Vol. 158, 2009, pp. 125-143.

${ }^{8}$ Bednarcyk, B. A. and Arnold, S. M., "MAC/GMC 4.0 User's Manual - Keywords Manual," NASA/TM 2002212077/VOL2, 2002.

${ }^{9}$ Bednarcyk, B. A. and Arnold, S. M., "MAC/GMC 4.0 User's Manual - Example Problems Manual," NASA/TM 2002212077/VOL3, 2002.

${ }^{10}$ Bednarcyk, B. A., Aboudi, J., and Arnold, S. M., "Micromechanics modeling of composites subjected to multiaxial progressive damage in the constituents," AIAA J., Vol. 48, 2010, pp. 1367-1378.

${ }^{11}$ Paley, M. and Aboudi, J., "Micromechanical Analysis of Composites by the Generalized Cells Model," Mechanics of Materials, Vol. 14, 1992, pp. 127-139.

${ }^{12}$ Moncada, A. M., Chattopadhyay, A., Bednarcyk, B. A., and Arnold, S. M., "Micromechanics-based progressive failure analysis of composite laminates using different constituent failure theories," J. Reinf. Plast. Comp., Vol. 31, No. 21, 2012, pp. 1467-1487.

${ }^{13}$ Pineda, E. J., Bednarcyk, B. A., Waas, A. M., and Arnold, S. M., "Progressive failure of a unidirectional fiber-reinforced composite using the method of cells: Discretization objective computational results," Int. J. Solids Struct., Vol. 50, No. 9, 2013, pp. 1203-1216.

${ }^{14}$ Pineda, E. J., Waas, A. M., Bednarcyk, B. A., Collier, C. S., and Yarrington, P. W., "A multiscale progressive damage and failure modeling approach for laminated fiber reinforced composites," Advances in Mathematical Modeling and Experimental Methods for Materials and Structures: The Jacob Aboudi Volume, edited by R. Gilat and L. Banks-Sills, Vol. 168 of Solid Mechanics and Its Applications, Springer, 2010, pp. 43-56.

${ }^{15}$ Pineda, E. J., A Novel Multiscale Physics-Based Progressive Damage and Failure Modeling Tool for Advanced Composite Structures, Ph.D. thesis, Universtiy of Michigan, Ann Arbor, MI, 2012.

${ }^{16}$ Pineda, E. J., Bednarcyk, B. A., Waas, A. M., and Arnold, S. M., "On multiscale modeling using the generalized method of cells: Preserving energy dissipation across disparate length scales," CMC-Comput. Mater. Con., Vol. 25, No. 2, 2013, pp. 119-154.

${ }^{17}$ Hashin, Z., "Failure criteria for unidirectional fiber composites," J. Appl. Mech., Vol. 47, No. 2, 1980, pp. $329-334$.

${ }^{18}$ Basu, S., Computational Modeling of Progrssive Failure and Damage in Composite Laminates, Ph.D. thesis, University of Michigan, Ann Arbor, MI, 2005.

${ }^{19}$ Pineda, E. J., Waas, A. M., Bednarcyk, B. A., and Collier, C. S., "Computational implementation of a thermodynamically based work potential model for progressive microdamage and transverse cracking in fiber-reinforced laminates," 51st AIAA/ASME/ASCE/AHS/ASC Structures, Structural Dynamics, and Materials Conference, Orlando, FL, 12-15 April 2010.

${ }^{20}$ Pineda, E. J. and Waas, A. M., "Modelling progressive failure of fibre reinforced laminated composites: mesh objective calculations," Aeronaut. J., Vol. 116, No. 1186, 2012, pp. 1221-1246.

${ }^{21}$ Pineda, E. J. and Waas, A. M., "Mesh Objective Implementation of a Thermodynamically-Based Work Potential Theory for Modeling Progressive Damage and Failure in Fiber-Reinforced Laminates," Int. J. Fract., Vol. 182, 2013, pp. 93-122.

${ }^{22}$ P. Davidson, E. J. Pineda, C. H. A. M. W., "A Unified model for predicting the open hole tensile and compressive strengths of composite laminates for aerospace applications," 54th AIAA/ASME/ASCE/AHS/ASC Structrues, Sturctural Dynamics, and Materials Conference, Boston, MA, 8-11, April 2013.

${ }^{23}$ Lamborn, M. J. and Schapery, R. A., "An investigation of deformation path-independence of mechanical work in fiberreinforced plastics," Proceedings of the Fourth Japan-U.S. Conference on Composite Materials, Technomic Publishing Co., Inc., Lancaster, PA, 1988.

${ }^{24}$ Lamborn, M. J. and Schapery, R. A., "An investigation of the existence of a work potential for fiber-reinforced plastic," J. Compos. Mater., Vol. 27, 1993, pp. 352-382.

${ }^{25}$ Lacy, Jr., T. E., Distribution Effects in Damage Mechanics, Ph.D. thesis, Georgia Institute of Technology, Atlanta, Georgia, 1998.

${ }^{26}$ Lacy, Jr., T. E., McDowell, D. L., and Talreja, R., "Gradient concepts for evolution of damage," Mech. Mater., Vol. 31, 1999, pp. 831-860. 